\title{
RENDIMENTO DE UVA 'NIAGARA ROSADA' SUBMETIDA À REDUÇÃO DE ÁREA FOLIAR ${ }^{1}$
}

\author{
JOÃO ALEXIO SCARPARE FILHO ${ }^{2}$, ANDRÉ LUIS DE MORAES ${ }^{3}$, \\ ALESSANDRO RODRIGUES ${ }^{4}$, FÁBIO VALE SCARPARE $^{5}$
}

RESUMO-O objetivo deste trabalho foi verificar o efeito da perda da área foliar da videira no período compreendido entre a colheita e a queda natural das folhas, sobre o rendimento das safras futuras Após colheita realizada em 15-12-2005, selecionaram-se plantas que apresentavam oito ramos de produção. Os tratamentos constituíram-se de plantas com duas intensidades de desfolhas artificiais $(25 \%$ e $50 \%)$, em quatro datas distintas $(30 ; 45 ; 60$ e 75 dias após a colheita - DAC), plantas sem redução artificial da área foliar, com proteção química (controle) e plantas sem redução artificial da área foliar e sem proteção química (testemunha). Dois ciclos de produção foram avaliados: ciclo da poda seca de julho/2006 com colheita em dezembro/2006 e ciclo da poda verde de fevereiro/2007 com colheita em junho/2007. Analisaram-se o crescimento de ramos, o número de cachos por planta e o rendimento $(\mathrm{kg} / \mathrm{planta})$ para os dois ciclos. Análises de regressão foram realizadas para as diferentes intensidades de redução de área foliar. A intensidade de desfolha após a colheita reduz o rendimento dos ciclos de produção futuros, porém não influencia no crescimento de ramos e no número de cachos produzidos na safra subsequente à redução da área foliar. $\mathrm{O}$ rendimento da safra da poda seca é maior do que a da poda verde.

Termos para indexação: Vitis labrusca L., desfolha, poda seca, poda verde.

\section{'NIAGARA ROSADA' GRAPEVINE YIELD UNDER DEFOLIATION}

\begin{abstract}
The objective of this work was to verify the effect of defoliation in 'Niagara Rosada' grapevine between harvest and natural leaf fall period and the influence on yield in the next cycle. After harvest, December-2005, were selected plants with eight production branches. The treatments were represented by plants under two artificial defoliation intensity $(25 \%$ and $50 \%)$ in four dates after harvest $(30,4560$ and 75), plants without artificial defoliation under chemical protection and plants without defoliation and without chemical protection. Two production cycles were appraised: dry pruning, from July-2006 until December-2006 (harvest) and green pruning, from February-2007 until June-2007 (harvest). Shots growth, number of bunches per plant and yield $\left(\mathrm{kg}\right.$.plant $\left.{ }^{-1}\right)$ were analyzed in both cycles. The regression analyses were done considering the intensity of defoliation. The intensity of defoliation after harvest reduces future yield for next cycles, however it does not show influence on shots growth and the number of bunches per plant. Dry pruning yield was bigger than green pruning yield.
\end{abstract}

Index terms: Vitis labrusca L., defoliation intensity, dry pruning, green pruning.

\section{INTRODUÇÃO}

A produção de uva no Brasil segue a tendência mundial que mostra uma especialização regional baseada em poucas variedades (VERDI et al., 2005). A videira 'Niagara Rosada' (Vitis labrusca L.) é a principal cultivar de uva rústica de mesa, sendo plantada principalmente nos Estados de São Paulo, Santa Catarina e Minas Gerais, representando aproximadamente $75 \%$ da produção e destacando-se como uma das uvas de mesa mais apreciadas pelo consumidor brasileiro (TERRA, 1996; POMMER et al., 1997; AGRIANUAL 2007).

Planta originária de clima temperado, a videira, após passar por um período de inatividade, caracterizado pelo repouso hibernal, inicia seu ciclo vegetativo. Autores como Hidalgo (2002), Pires e Pommer (2003) e Reynier (2003) definem-no de acordo com as seguintes fases: mobilização das substâncias de reserva, crescimento dos órgãos da

\footnotetext{
'(Trabalho 182-09). Recebido em: 06-08-2009. Aceito para publicação em: 28-01-2010.

${ }^{2} E^{\circ}{ }^{\circ}$. Agr. Professor Doutor Associado - Depto de Produção Vegetal da ESALQ/USP, Av. Pádua Dias 11, Cx. Postal 09, CEP: 13.418900 Piracicaba-SP - jascarpa@esalq.usp.br

${ }^{3} E_{n g}$. Agr., MSc, Gerente de Tecnologia - Dupont de Colômbia S.A., Calle 113 No 7-21, Torre A Piso 14, Bogotá - Colômbia andre-luis.moraes@bra.dupont.com

${ }^{4} E_{n g}$. Agr. Doutor em Fitotecnia - Rua Piracicaba, 50 - Artemis - CEP: 13.432-021 - Piracicaba-SP -rodriguesale@yahoo.com.br ${ }^{5}$ Eng $^{\circ}$.Agrônomo MSc., Doutorando em Física do Ambiente Agrícola da ESALQ/USP - Av. Pádua Dias 11, Cx. Postal 09, CEP: 13418900 Piracicaba-SP - fvscarpa@esalq.usp.br
} 
planta e acúmulo de reservas. A fase de acúmulo de reservas dá-se com a maturação dos frutos e termina com a queda natural das folhas (HIDALGO, 2002). Após a colheita, a maioria dos fotossintetizados move-se do ramo para outras partes da planta, sendo os açúcares convertidos em amido nas partes permanentes da videira (tronco, braços, raízes). Todos os tecidos lenhosos de armazenamento constituem drenos nessa época, desempenhando as raízes o papel principal (SCHIEDECK, 1996). Para iniciar novo ciclo vegetativo, o amido é convertido em açúcares, que serão utilizados para nutrirem as novas brotações (KLIEWER, 1990 GIOVANNINI, 1999; BATES et al., 2002).

No Estado de São Paulo, a videira é tradicionalmente podada após o repouso hibernal. Esta poda é realizada no fim do inverno, conhecida como poda seca e que atinge o período de colheita em pleno verão (dezembro/janeiro). Uma segunda colheita entre os meses de maio e junho é possível quando efetuada a poda verde, realizada no segundo fio da espaldeira, nos meses de janeiro e fevereiro. Scarpare Filho e Watanabe (2004) ressaltam que a poda verde não deve ser realizada todo ano na mesma área de plantio, evitando que a planta apresente alternância de safras, sendo as causas mais prováveis a redução do período de acúmulo de reservas e o esgotamento das reservas dos órgãos perenes.

A desfolha é uma ferramenta eficaz que altera o balanço entre armazenamento e dreno das reservas. Dados experimentais indicam que a diminuição da área foliar realizada pela prática da desfolha aumenta a taxa fotossintética em folhas remanescentes, estimulando a assimilação dos fotoassimilados (CANDOLFI-VASCONCELOS et al., 1994). Autores como Hunter et al. (1995) comentam que desfolhas parciais são usadas com o objetivo de promover melhoras na parte aérea das videiras, além de incrementar o rendimento. Porém, segundo Bennett et al. (2005), a diminuição da área foliar pode restringir o acúmulo de carboidratos, tendo como consequências impactos negativos no florescimento e no rendimento.

O estudo da manutenção da área foliar no cultivo da videira mostra-se pertinente devido à larga utilização da prática de poda de produção e por apresentar grande importância em regiões onde alta umidade relativa do ar propicia ambiente ideal para a proliferação de fungos que causam a desfolha natural (CHANISHVILI et al., 2005).

O objetivo deste trabalho foi verificar o efeito da perda da área foliar da videira, no período compreendido entre a colheita e a queda natural das folhas, sobre o rendimento das safras futuras.

\section{MATERIAL E MÉTODOS}

O experimento foi instalado em 15 de dezembro de 2005, no município de Indaiatuba-SP (latitude $23^{\circ} 05^{\prime}$ sul e longitude $47^{\circ} 13^{\prime}$ oeste). O tipo climático da região, segundo a classificação de Köeppen, é Cwa (tropical de altitude), com três meses mais secos (junho/julho/agosto), chuvas predominantes de verão e seca no inverno. A temperatura média no mês mais quente é maior que $22^{\circ} \mathrm{C}$, e a do mês mais frio, menor que $18^{\circ} \mathrm{C}$, com média de $21,1^{\circ} \mathrm{C}$, precipitação média de $1.253 \mathrm{~mm} /$ ano e umidade relativa do ar de $74 \%$. $\mathrm{O}$ solo da área experimental é classificado como Latossolo Vermelho-Amarelo (EMBRAPA, 1999). Os resultados da análise química de solo podem ser visualizados na Tabela 1.

O experimento foi constituído de 120 plantas de videira 'Niagara Rosada' enxertadas sobre portaenxerto 'Ripária do Traviú', em vinhedo comercial de três anos de idade, conduzido no sistema de espaldeira com duplo cordão esporonado, em espaçamento de 1,0 m X 1,7 m, correspondendo a 5.882 plantas por hectare. Essas plantas haviam sido podadas em julho de 2005 (poda seca), com colheita realizada em 15-12-2005.

Após a colheita, selecionaram-se plantas que apresentavam oito ramos de produção. Para verificar a perda de área foliar, causada normalmente pela incidência de doenças fúngicas no período de acúmulo de reservas, estabeleceram-se tratamentos com duas intensidades de desfolhas artificiais $(25 \% \mathrm{e}$ $50 \%)$ em quatro datas distintas $(30 ; 45 ; 60$ e 75 dias após a colheita-DAC), plantas sem redução artificial da área foliar com proteção química (controle) e plantas sem redução artificial da área foliar e sem proteção química (testemunha) (Tabela 2).

O controle fitossanitário de doenças foi realizado até a queda natural das folhas, visando principalmente ao controle preventivo do míldio (Plasmopara viticola) e da Mancha da Folha (Isariopsis clavispora), por meio de aplicações dos fungicidas que possuem os seguintes princípios ativos: (famoxadone $22,5 \%+$ cymoxanil $30 \%$ ) e (difeconazole 25\%).

Foram avaliados dois ciclos de produção subsequentes aos tratamentos de desfolha: a poda seca com uma gema, realizada no mês de julho de 2006, com colheita em dezembro de 2006 e a poda verde com cinco gemas (poda do segundo fio de arame), realizada no mês de fevereiro de 2007 e com colheita em junho de 2007.

As variáveis analisadas após a poda seca de julho de 2006 foram: comprimento de ramos 
realizado semanalmente, a partir da brotação das gemas até 56 dias após a poda; número de cachos formados por planta, e rendimento ( $\mathrm{kg} /$ planta). As variáveis analisadas após a poda verde de fevereiro de 2007 foram: comprimento de ramos realizado semanalmente a partir da brotação das gemas até 35 dias após a poda; número de cachos formados por planta, e rendimento ( $\mathrm{kg} /$ planta). A medição de ramos foi realizada com auxílio de uma fita métrica graduada em cm, avaliando-se dois ramos da planta central de cada parcela, totalizando 80 ramos.

A taxa de crescimento absoluta de ramos (TCA), em $\mathrm{cm} /$ dia para as duas épocas de poda, foi calculada pela seguinte fórmula:

$\mathrm{TCA}=($ comprimento final - comprimento inicial) / (tempo final - tempo final)

O delineamento estatístico utilizado foi o de blocos casualizados, com 10 tratamentos e 4 repetições, sendo três plantas por parcela. Os resultados foram submetidos à análise de variância, e a comparação das médias, pelo teste de Duncan, ao nível de $5 \%$ de probabilidade. Os resultados de rendimentos obtidos nas diferentes intensidades de desfolha foram submetidos à análise de regressão polinomial.

\section{RESULTADOS E DISCUSSÃO}

O crescimento e a taxa de crescimento de ramos, em ambas as podas (seca e verde), não foram afetados pelos tratamentos que reduziram a área foliar na fase de acúmulo de reservas. Ambos apresentaram um crescimento sigmoidal, confirmando os resultados obtidos por diversos autores como Hidalgo (2002), Reynier (2003) e Scarpare et al. (2007), que descrevem um crescimento lento no início do desenvolvimento dos ramos, passando por um período acelerado (quase um crescimento vertical), seguido por um ritmo desacelerado de crescimento.

Na poda seca, a taxa de crescimento absoluta de ramos alcançou seu ápice em torno de 30 dias $(4,5 \mathrm{~cm} / \mathrm{dia})$, e foi diminuindo gradativamente até o florescimento. Pode-se observar, na Figura 1A, que os ramos nesse ciclo alcançaram em média um comprimento de 100 centímetros, atingindo seu máximo de comprimento, 56 dias após a poda.

Aos 20 dias após a poda verde, a taxa de crescimento absoluta de ramos alcançou o seu ápice $(2,6 \mathrm{~cm} / \mathrm{dia})$, e os ramos atingiram seu máximo comprimento, aproximadamente $50 \mathrm{~cm}$, aos 35 dias após a poda verde (Figura 1B).

Analisando o crescimento de ramos após a poda verde em comparação com a poda seca, verificaram-se menor crescimento, menor tempo para atingir seu máximo comprimento e menor taxa de crescimento de ramos. Esses resultados são semelhantes aos obtidos por Scarpare Filho e Watanabe (2004), que, trabalhando com uva 'Niagara Rosada' no município de Atibaia-SP, verificaram que o tamanho dos ramos da poda seca foi, em média, 95 centímetros, enquanto na poda verde, 58 centímetros.

Não houve influência da diminuição da área foliar sobre o número de cachos produzidos por planta durante o ciclo da poda seca (Tabela 3). Isso era esperado, pois, de acordo com Kliewer (1990), os rudimentos ou primórdios das inflorescências de videira são formados durante a estação que precede o ano no qual as flores vão surgindo. Se um ramo terá ou não cacho na safra seguinte, isso já foi determinado no ciclo de produção anterior. Essa determinação ocorre precocemente durante a formação da gema. Existem indicações de que a indução de células para primórdio de cacho ocorre nas primeiras semanas depois que um nó se separa de um ápice. Como o experimento foi instalado em meados do verão, as gemas que formariam os cachos já estavam determinadas. No entanto, no ciclo da poda verde, houve interferência dos tratamentos na formação de cachos. A menor formação de cachos contribuiu para a redução no rendimento do ciclo da poda verde. Todavia, o tratamento-controle mostrouse superior à testemunha (Tabela 3).

$\mathrm{O}$ rendimento em $\mathrm{kg} /$ planta para o ciclo da poda seca foi afetado pela diminuição da área foliar. $\mathrm{O}$ tratamento sem desfolha artificial e sem controle fitossanitário (testemunha) apresentou redução de área foliar causada pela incidência de doenças fúngicas, resultando em menor rendimento $(2,37$ $\mathrm{kg} /$ planta), o que representa $55 \%$ do rendimento obtido no tratamento sem desfolha e com controle fitossanitário (controle) (Tabela 3).

Os resultados obtidos neste trabalho demonstraram que o controle das doenças fúngicas na fase de acúmulo de reservas é de extrema importância para se evitar uma perda precoce das folhas. Além de as doenças causarem redução na área foliar, $\mathrm{Su}$ tic e Sinclair (1991) observaram que as alterações fisiológicas sofridas pelas plantas doentes são muito severas, levando a um gasto excessivo de energia para poder combatê-las, o que reflete num menor rendimento para o ciclo posterior. Bledsoe (1988), Pedro Júnior et al. (1992) e Candolfi-Vasconcelos et al. (1994), realizando trabalhos de desfolha com uva, também observaram que os tratamentos que não recebiam nenhuma desfolha mantinham maior rendimento.

As análises de regressão polinomial para os 
diferentes ciclos e intensidades de desfolha podem ser visualizadas na Tabela 4.

Observa-se que houve forte correlação, principalmente para intensidade de $50 \%$ de redução da área foliar no ciclo da poda seca. Porém, o rendimento do ciclo da poda verde apresentou fraca correlação para as diferentes intensidades de redução de área foliar.

A hipótese esperada era que, quanto mais precoce e intensa fosse a redução da área foliar em plantas de videira, maiores seriam os prejuízos no rendimento das safras futuras. No entanto, a análise de regressão para o primeiro ciclo (poda seca) mostrou uma tendência contrária, ou seja, as menores colheitas foram observadas nas desfolhas tardias e mais intensas, com exceção da redução precoce de $25 \%$ da área foliar, realizada aos 30 DAC (Figura 2A).
Embora tendo menor rendimento no segundo ciclo após a instalação dos tratamentos (poda verde), plantas submetidas a 50\% de redução de área foliar apresentaram uma tendência de alternância de safras, ou seja, houve inversão quando comparadas com a safra anterior (Figura 2B). Possivelmente, isso tenha ocorrido devido ao maior dispêndio de energia necessária ao desenvolvimento e à produção do ciclo anterior. Porém, para as plantas que foram submetidas à redução de $25 \%$ de sua área foliar, observou-se comportamento semelhante ao da safra da poda seca, ou seja, os maiores prejuízos foram observados na redução da área foliar, precoce e tardia.

TABELA 1 - Resultado da análise química do solo da área experimental. Indaiatuba, 2006.

\begin{tabular}{cccccccc}
\hline $\begin{array}{c}\mathbf{p H} \\
\mathrm{CaCl} 2\end{array}$ & $\begin{array}{c}\mathbf{M . O} \\
\text { g.dm }\end{array}$ & $\mathbf{S}$ & $\mathbf{P}$ & $\mathbf{K}$ & $\mathbf{C a}$ & $\mathbf{M g}$ & $\mathbf{V}$ \\
\hline 5,9 & 12 & 7 & 68 & 1,2 & 34 & 9 & 77 \\
\hline
\end{tabular}

TABELA 2 - Tratamentos realizados com e sem redução da área foliar e controle fitossanitário após a colheita de uva 'Niagara Rosada'. Indaiatuba, 2006.

\begin{tabular}{lcc}
\hline $\begin{array}{c}\text { Tratamentos (\% de redução da área foliar) e } \\
\text { (data da realização) }\end{array}$ & $\begin{array}{c}\text { Dias após a } \\
\text { colheita (DAC) }\end{array}$ & $\begin{array}{c}\text { Controle } \\
\text { fitossanitário de } \\
\text { doenças }\end{array}$ \\
\hline T1: (0\%) (controle) & - & Sim \\
T2: (25\%) (15/01/06) & 30 & Sim \\
T3: (50\%) (15/01/06) & 30 & Sim \\
T4: (25\%) (30/01/06) & 45 & Sim \\
T5: $(50 \%)(30 / 01 / 06)$ & 45 & Sim \\
T6: $(25 \%)(15 / 02 / 06)$ & 60 & Sim \\
T7: $(50 \%)(15 / 02 / 06)$ & 60 & Sim \\
T8: $(25 \%)(02 / 03 / 06)$ & 75 & Sim \\
T9: $(50 \%)(02 / 03 / 06)$ & 75 & Sim \\
T10: $(0 \%)$ (testemunha) & - & Não \\
\hline
\end{tabular}


TABELA 3 - Rendimento de uva 'Niagara Rosada' (número de cachos/planta e kg/planta) nos ciclos de poda seca e de poda verde ${ }^{(1)}$. Indaiatuba, 2006/2007.

\begin{tabular}{ccccc}
\hline $\begin{array}{c}\text { Tratamentos (\% de redução } \\
\text { da área foliar) e (DAC }=\text { dias } \\
\text { após a colheita) }\end{array}$ & $\begin{array}{c}\text { PuDA SECA } \\
\text { planta }\end{array}$ & $\begin{array}{c}\text { PODA VERDE } \\
\text { (kg/planta) }\end{array}$ & $\begin{array}{c}\text { Púmero cachos/ } \\
\text { planta }\end{array}$ & $\begin{array}{c}\text { Rendimento } \\
\text { (kg/planta) }\end{array}$ \\
\hline 1: (0\%) (controle) & $12,00 \mathrm{a}$ & $4,25 \mathrm{a}$ & $10,00 \mathrm{abc}$ & $1,93 \mathrm{a}$ \\
2: $(25 \%)(30 \mathrm{DAC})$ & $11,25 \mathrm{a}$ & $2,78 \mathrm{bc}$ & $8,33 \mathrm{bcd}$ & $1,22 \mathrm{~b}$ \\
3: $(50 \%)(30 \mathrm{DAC})$ & $11,75 \mathrm{a}$ & $3,43 \mathrm{abc}$ & $8,33 \mathrm{bcd}$ & $1,33 \mathrm{~b}$ \\
$\mathbf{4 :}(25 \%)(45 \mathrm{DAC})$ & $10,75 \mathrm{a}$ & $3,55 \mathrm{abc}$ & $10,67 \mathrm{a}$ & $1,92 \mathrm{a}$ \\
$\mathbf{5 :}(50 \%)(45 \mathrm{DAC})$ & $12,25 \mathrm{a}$ & $3,12 \mathrm{abc}$ & $9,00 \mathrm{abcd}$ & $1,79 \mathrm{ab}$ \\
$\mathbf{6}:(25 \%)(60 \mathrm{DAC})$ & $13,50 \mathrm{a}$ & $4,04 \mathrm{ab}$ & $8,33 \mathrm{bcd}$ & $1,53 \mathrm{ab}$ \\
7: $(50 \%)(60 \mathrm{DAC})$ & $10,00 \mathrm{a}$ & $2,81 \mathrm{bc}$ & $10,33 \mathrm{ab}$ & $1,59 \mathrm{ab}$ \\
$\mathbf{8 :}(25 \%)(75 \mathrm{DAC})$ & $11,50 \mathrm{a}$ & $2,85 \mathrm{bc}$ & $10,33 \mathrm{ab}$ & $1,32 \mathrm{~b}$ \\
$\mathbf{9 :}(50 \%)(75 \mathrm{DAC})$ & $12,25 \mathrm{a}$ & $2,80 \mathrm{bc}$ & $8,00 \mathrm{~cd}$ & $1,65 \mathrm{ab}$ \\
$\mathbf{1 0}:(0 \%)$ (testemunha) & $10,75 \mathrm{a}$ & $2,37 \mathrm{c}$ & $7,67 \mathrm{~d}$ & $1,27 \mathrm{~b}$ \\
\hline CV (\%) & 26,64 & 26,76 & 12,2 & 19,43 \\
\hline
\end{tabular}

(1) Médias seguidas de mesma letra na coluna não diferem entre si, pelo teste de Duncan $(\mathrm{P} \leq 5 \%)$.

TABELA 4 - Análises de regressão polinomial e coeficientes de correlação para diferentes ciclos de produção e intensidades de redução de área foliar após a colheita de uva 'Niagara Rosada'. Indaiatuba $2006 / 2007$

\begin{tabular}{lccc}
\hline Safra & \% de redução da área foliar & Regressão polinomial & $\begin{array}{c}\text { Coeficiente de correlação } \\
\left(\mathbf{R}^{2}\right)\end{array}$ \\
\hline Poda seca & $25 \%$ & $-0,0022 \mathrm{x}^{2}+0,2333 \mathrm{x}-2,33$ & 0,9095 \\
Poda seca & $50 \%$ & $0,0003 \mathrm{x}^{2}+0,0497 \mathrm{x}+4,635$ & 0,9833 \\
Poda verde & $25 \%$ & $-0,001 \mathrm{x}^{2}+0,1056 \mathrm{x}-0,9735$ & 0,7201 \\
Poda verde & $50 \%$ & $-0,0004 \mathrm{x}^{2}+0,0517 \mathrm{x}+0,224$ & 0,6194 \\
\hline
\end{tabular}

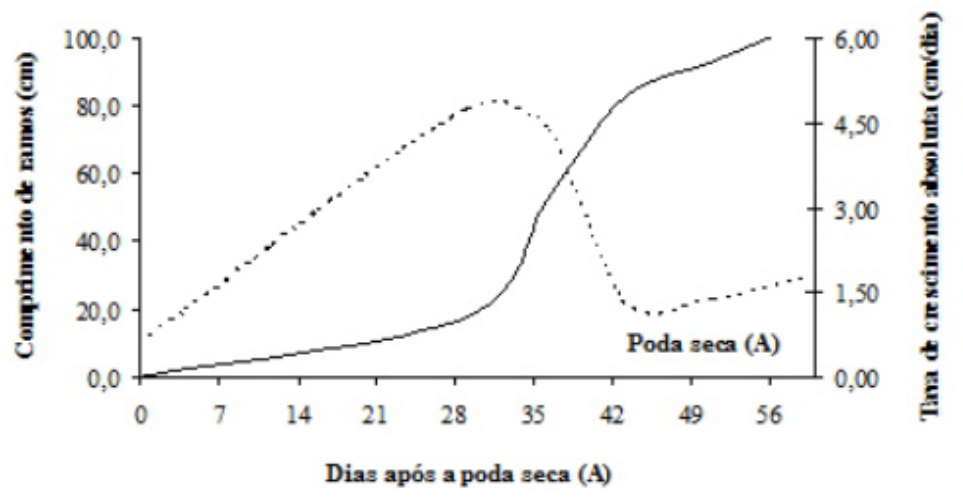




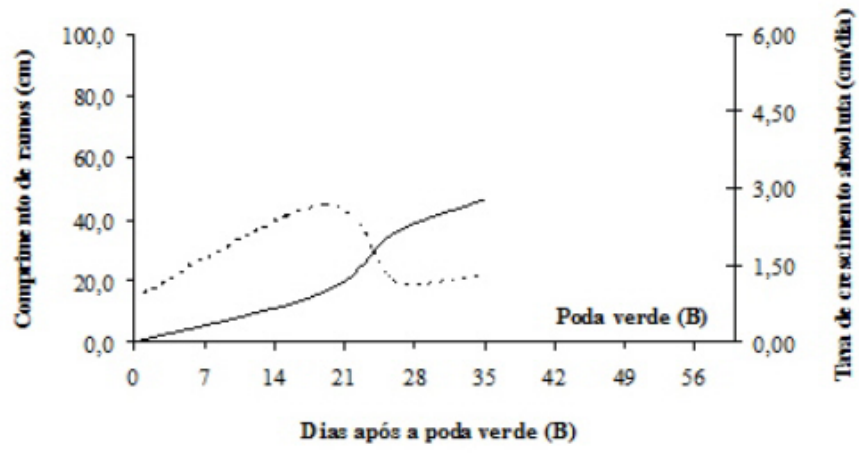

\section{--- Taxa de crescim ento absoluta}

FIGURA 1 - Comprimento (cm) e taxa de crescimento absoluta ( $\mathrm{cm} /$ dia) de ramos de videira 'Niagara Rosada' após a poda seca (A) e após a poda verde (B). Indaiatuba 2006/2007.

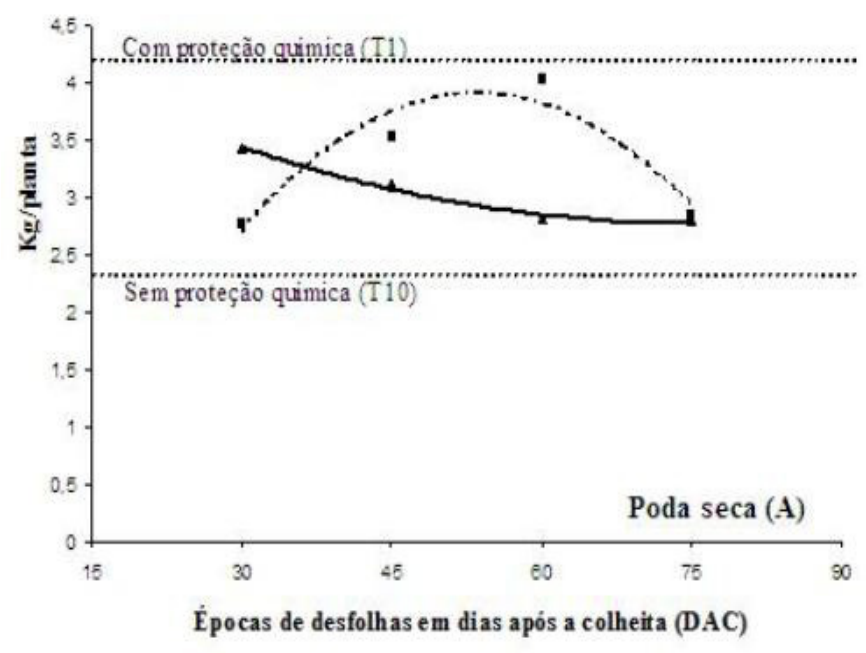

- $50 \%$ de redução de área foliar

continua 


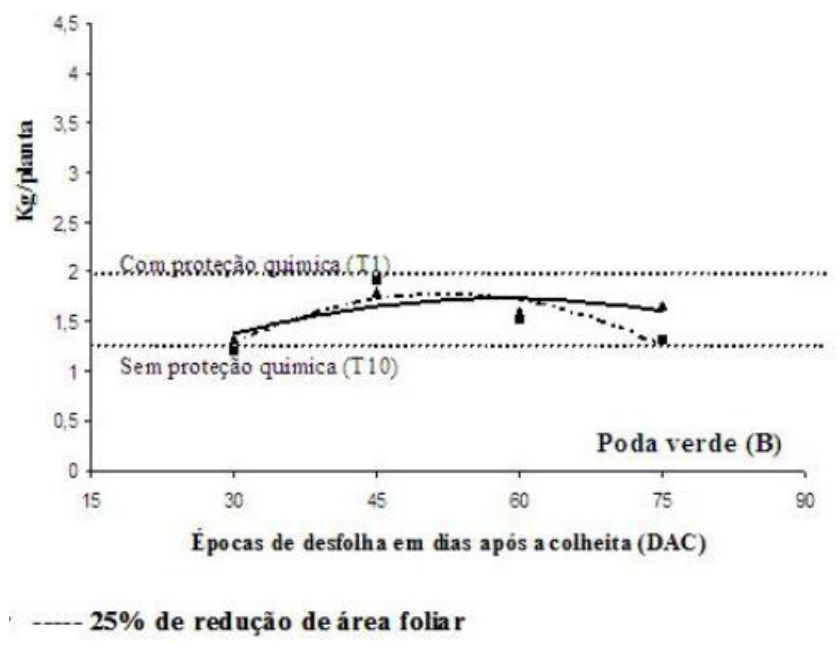

FIGURA 2 - Rendimento de uvas 'Niagara Rosada' (kg/planta) no ciclo da poda seca (A) e poda verde (B), com e sem proteção química na fase de acúmulo de reservas para redução da área foliar, em diferentes datas e intensidades após a colheita. Indaiatuba, 2006/2007.

\section{CONCLUSÃO}

A intensidade de desfolha em videira, após a colheita, reduz o rendimento dos ciclos de produção futuros, porém não influencia no crescimento de ramos e no número de cachos produzidos na safra subsequente à redução da área foliar. O rendimento da safra da poda seca é maior do que o da poda verde.

\section{REFERÊNCIAS}

AGRIANUAL 2008: anuário estatístico da agricultura brasileira. São Paulo: Fnp Consultoria \& Comércio, 2007. 502 p.

BATES, T.R.; DUNST, R.M.; JOY, P. Seasonal dry matter, starch, and nutrient distribution in 'Concord' grapevine roots. HortScience, Alexandria, v.37, n.2, p.313-316, 2002.

BENNETT, J.; JARVIS, P.; CREASY, G.L.; TROUGHT, M.C.T. Influence of defoliation on overwintering carbohydrate reserves, return bloom and yield of mature Chardonnay grapevines. American Journal of Enology and Viticulture, Davis, v.56, n.4, p.386-393, 2005.
BLEDSOE, A.M.; KLIEWER, W.M.; MAROIS, J.J. Effects of timing and severity of leaf removal on yield and fruit composition of sauvignon blanc grapevines. American Journal of Enology and Viticulture, Davis, v.39, n.1, p.49-54, 1988.

CANDOLFI-VASCONCELOS, M.C.; KOBLET, W.; HOWELL, G.S.; ZWEIFEL.W. Influence of defoliation, rootstock, training system, and leaf position on gas exchange of Pinot noir grapevines. American Journal of Enology and Viticulture, Davis, v.45, n.2, p.173-180, 1994.

CHANISHVILI, Sh. Sh.; BADRIDZE,G. Sh.; BARBLISHVILI, T.F.; DOLIDZE, M.D. Defoliation, photosynthetic rates, and assimilate transport in grapevine plants. Russian Journal of Plant Physiology, Moscow, v.52, n.4, p.448-453, 2005.

\section{EMBRAPA. Sistema brasileiro de classificação} de solos. Brasília, 1999. 412 p.

GIOVANNINI, E. Produção de uvas para vinho, suco e mesa. Porto Alegre: Renascença, 1999. 364p.

HIDALGO, L. Tratado de viticultura general. 3 . ed. Madrid: Mundi-Prensa, 2002. 1235p. 
HUNTER, J.J.; RUFFNER, H.P.; VOLSCHENK, G.G.; LE ROUX, D.J. Partial Defoliation of Vitis vinifera L. cv. Cabernet Sauvignon/99 richter: effect on root growth, canopy efficiency; grape composition, and wine quality. American Journal of Enology and Viticulture, Davis, v.46, n.3, p.306-314, 1995.

KLIEWER, W.M. Fisiologia da videira: como produz açúcar uma videira? Trad. C.V. Pommer e I.R.S. Passos. Campinas: IAC, 1990. 51 p. (Documento Técnico, 20).

PEDRO JÚNIOR, M.J.; POMMER, C.V.; MARTINS, F.P.; RIBEIRO, I.J.A. Influência da diminuição da área foliar na produtividade e na duração do ciclo da videira 'Niagara Rosada' Bragantia, Campinas, v.51, n.1, p.57-61, 1992.

PIRES, E.J.P.; POMMER, C.V. Fisiologia da videira. In: POMMER, C.V. Uva: tecnologia de produção, pós-colheita, mercado. Porto Alegre: Cinco Continentes, 2003. p.250-294.

POMMER, C. V.; PASSOS, I.R.S.; TERRA, M.M.; PIRES, E.J.P. Variedades de videira para o Estado de São Paulo. Campinas: IAC, 1997. 59 p. (Boletim Técnico, 166).

REYNIER, A. Manual de viticultura. 6.ed. Madrid: Mundi-Prensa, 2003. 497 p.

SCARPARE FILHO, J. A.; WATANABE, A. T. Relação entre os teores de carboidratos solúveis em raízes e os estádios fenológicos, em dois ciclos de produção. In: SIMPÓSIO DE VITICULTURA DO ALENTEJO, 6., 2004, Évora. Actas... Évora: ATEVA; CCDRA; CVRA; DRAAI; EU, 2004. p. 199-209.
SCARPARE, F.V.; ANGELOCCI, L.R.; SCARPARE FILHO, J.A.; SIMON, J.; SENTELHAS, P.C.; CASAROLI, D. Graus-dia relacionado com o crescimento de ramos da videira 'Niagara Rosada'. In: CONGRESSO BRASILEIRO DE AGROMETEOROLOGIA, 15., 2007, Aracaju. Anais ... Aracaju: Sociedade Brasileira de Agrometeorologia, 2007. 1 CD-ROM.

SCHIEDECK, G. Ecofisiologia da videira e qualidade da uva 'Niagara Rosada' conduzida sob estufa de plástico. 1996. 112 f. (Mestrado em Fitotecnia) - Universidade Federal do Rio Grande do Sul, Porto Alegre, 1996.

SUTIC, D.D.; SINCLAIR, J.B. Physiology of diseased plants. In: SUTIC, D.D.; SINCLAIR, J.B. Anatomy and physiology of diseased plants. Boca Raton: CRC Press, 1991.p. 157-222.

TERRA, M. M. A produção de uvas no sul do Estado de Minas Gerais. In: ENCONTRO SUL MINEIRO DE FRUTICULTURA DE CLIMA TEMPERADO, 1., 1996, Poços de Caldas. Anais... p. 12-22.

VERDI, A.R.; SILVA, P.R.; FRANCISCO, V.L.F.S.; AMARO, A.A.; BAPTISTELLA, C.S.L. Arranjo produtivo local: identificação das possibilidades da viticultura da região de Campinas. Revista Agricultura em São Paulo, São Paulo, v.52, n.2, p.73-86, 2005 . 